themselves. In the long run it would be a great advantage if British universities had more money to call their own (and they could do a lot to help themselves by playing on the affections of their alumni), but there is no realistic prospect that the universities will be able to buy their way out of trouble.

In any case, the weakness of the universities is partly their own creation. They are too vulnerable to sharp questioning about cost-effectiveness. Although some of the newer universities have taken it on themselves to carry out deliberate studies of the most efficient ways of teaching, many institutions are unreasonably sensitive to suggestions that different ways might bring greater benefits. Ironically enough, teachers as well as students often suffer from the same mismanagement. In Oxford, for example, the Franks Report-as much a defence of the present arrangements for undergraduate education as a proposal for reform -showed quite clearly how strictly administrative changes could bring great benefits in the working of the university. Sir Hans Krebs, in his article in this issue (see page 1441), shows how the same muddles impede the conduct of research at Oxford. So is it surprising that the custodians of the public purse should have become uncomfortably suspicious of the way the universities conduct their business? And because there is no prospect that British universities will ever be entirely free from dependence on public funds, is it realistic of them to seck autonomy without first taking scrupulous care to see that their own operations are above criticism of all kinds? In the long run, the universities will only be able to defend themselves against intrusion when they are better able than their would-be critics to decide what part universities should play in modern society and then to decide how best to attain desirable objectives. This means in practice that the universities must organize themselves for change. Somehow they must design a means by which they can arrive, by common consent, at workable decisions.

Strengthening the Committee of Vice-Chancellors is an obvious step to take, but means will also have to be found to give the people who work in universities a better sense of being represented and a clearer conviction that they must be bound by collective decisions. Inevitably, universitics will have to become more attentive to problems of cost-effectiveness. They need to be so enterprising in this respect that they can forestall the criticisms of those outside. When they have done everything that can be done in ways like these, it will be time enough to pass the hat around.

\section{TOO MUCH IRON?}

For several decades it has been plain that there are scrious problems to be solved before the abundance of iron in the parts of the universe accessible to observation can be properly accounted for. The most conspicuous difficulty to be overcome is that iron seems to be relatively much less abundant in the atmosphere of the Sun than in the solid objects of the Solar System - the metallic parts of the chondrites which reach the Earth as meteorites and the Earth itself. The article by P. G. Harris and D. C. Tozer on page 1449 of this issue is an ingenious attempt to account for what seems to be a segregation of iron into the substance of the inner planets. It is, of course, improbable that doubts will now be resolved-the authors would probably be the first to admit as much. But there is at least a chance that interest will be re-awakened in this awkward problem, which can only be a benefit.

The first thing to be said is that the problem of iron in the Solar System is almost the personal creation of Professor Harold Urey, who with his collaborators over several decades has compiled the evidence which shows the disconcerting variation of the abundance of iron from one object to another. The ratio of iron to silicon in the atmosphere of the Sun is less than a third of that in the chondrites, and only a fifth of the ratio of iron to silicon which must be assumed if the Earth has a molten core of iron and nickel of much the same composition as the metallic parts of meteorites. It is true that the relative abundance of iron on the Earth is comparable with that in the carbonaceous chondrites, which by this test are the most likely representatives of primeval matter, but this coincidence only makes it more necessary to explain why the Sun appears to be deficient in iron. Are the measurements in error? Or was there some process of fractionation during the formation of the Solar System which concentrated segregated iron in the inner planets?

Harris and Tozer have suggested a mechanism of the second kind. They suggest that iron may have accumulated preferentially in the inner plancts as a result of magnetic attractions between particles of iron dust in the material left over after the formation of the Sun. The point is that particles of iron held magnetically together are less likely than other kinds of dust to be swept away by the outward radiation pressure of the Sun. One difficulty, of course, is that it will only be possible to test this argument when much more is known about the details of the formation of the planets and about the mechanisms by means of which their composition has been altered in the past five thousand million years or so. Another is that of accepting such a comparatively simple explanation for what has secmed, in recent years, to be an extremely complicated problem. In the long run it is probable that room will have to be found in any comprehensive theory for at least some of the explanations which Urey has devised to explain, for example, how some kinds of meteorites may have lost some kinds of elements preferentially. Indeed, the complications of these arguments have at least the virtue that they bring together several different kinds of observations - the compositions of meteorites, the geophysics of the planets and the theoretical calculations of the natural abundances of the elements, for example. But none of this implies that there is no room for new ideas. Here, as elsewhere, every innovation helps. 